\title{
Rats' midsession reversal performance: the nature of the response
}

\author{
Aaron P. Smith ${ }^{1} \cdot$ Kristina F. Pattison ${ }^{1}$ - Thomas R. Zentall ${ }^{1,2}$
}

Published online: 23 July 2015

(C) Psychonomic Society, Inc. 2015

\begin{abstract}
The midsession reversal task involves a simple simultaneous discrimination that predictably reverses midway through a session. Under various conditions, pigeons generally both anticipate the reversal and perseverate once it has occurred, whereas rats tend to make very few of either kind of error. In the present research, we investigated the hypothesis that the difference in performance between rats and pigeons is related to the nature of the responses made. We hypothesized that rats could have been better at bridging the intertrial interval by keeping the relevant paw close to the lever while eating, whereas the pigeons had to remove their beak from the response key and insert it into the feeder, thus making it difficult to mediate the response last made. In the present experiment, in successive phases, rats were trained to leverpress or nose-poke on a 40-trial midsession reversal, an 80-trial midsession reversal, and a variable-location reversal. The results showed that the leverpress group acquired the task faster than the nose-poke group, but that both groups reached comparable levels of performance. Thus, the difference in the natures of the responses cannot fully account for the differences in accuracy between rats and pigeons. Additionally, differences in the types of errors made by the two groups suggest that the nature of the response plays different roles in the performance of this task.
\end{abstract}

Thomas R. Zentall

zentall@uky.edu

1 University of Kentucky, Lexington, KY, USA

2 Department of Psychology, University of Kentucky, Lexington, KY 40506-0044, USA
Keywords Midsession reversal · Discrimination · Anticipatory errors $\cdot$ Perseverative errors $\cdot$ Species differences $\cdot$ Win-stay/lose-shift $\cdot$ Reversal $\cdot$ Rats

The ability of an individual to flexibly change its behavior when environmental contingencies change is a skill with adaptive value (Shettleworth, 2010). Experimentally, learning to be flexible, or learning to learn, has often been assessed through the use of tasks that involve multiple sequential discriminations between two stimuli, positive $(\mathrm{S}+)$ and negative (S-), in which only responses to the $\mathrm{S}+$ are rewarded. For example, in learning-set tasks, discriminations are acquired involving novel pairs of stimuli that change from problem to problem. The measure of the learning set is the improvement in discrimination accuracy following feedback from the first trial with novel stimuli (e.g., Braggio, Braggio, Cochran, \& Ellen, 1983; Harlow, 1950). In reversal tasks, however, a single pair of stimuli is used and, at some point in training, the $\mathrm{S}+$ becomes the $\mathrm{S}-$ and the $\mathrm{S}-$ becomes the $\mathrm{S}+$. Typically, the task involves multiple reversals (serial reversal tasks; e.g., Bitterman, 1965) and the variable of interest is the reduction in errors with successive reversals or the improvement in reversal performance relative to the original discrimination (Rajalakshmi \& Jeeves, 1965). This reduction in errors has been characterized as a measure of intelligence (Bitterman, 1965 ) and as a measure of the ability to refrain from impulsive reward-seeking behaviors (see Izquierdo \& Jentsch, 2012, for a review).

A variation on the serial reversal task is the midsession reversal, in which the reversal occurs at a predictable point in the session (Rayburn-Reeves, Molet, \& Zentall, 2011; Rayburn-Reeves, Stagner, Kirk, \& Zentall, 2013; Stagner, Michler, Rayburn-Reeves, Laude, \& Zentall, 2013). Rayburn-Reeves et al. (2011) first trained pigeons on a simple 
simultaneous visual discrimination in which one stimulus, S1, was always correct for the first 40 trials, and the other stimulus, S2, was always correct for the remaining 40 trials of each session. After many sessions of training, the pigeons had a strong tendency to respond to S2 as the reversal point approached (anticipatory errors), and they continued to respond to S1 for several trials after the reversal had occurred (perseverative errors). It appeared that correct responding to the two stimuli was controlled by an estimate of the passage of time from the start of the session and took the form of a sigmoidal or ogive-shaped psychophysical function, similar to what has been found in a temporal bisection task (Platt \& Davis, 1983).

Since then, strong evidence has been found that pigeons do appear to use the passage of time as a cue to reverse (McMillan $\&$ Roberts, 2012, 2015). The use of time as a cue for reversing is not optimal, however, because judgments of time into the session provide a less accurate cue than the outcome of the preceding trial. Use of the outcome of the preceding trial as a cue to reverse can be described as a win-stay/lose-shift rule, which involves continued responding to the first correct stimulus (S1) until reinforcement does not follow, after which responding should switch to the other stimulus (S2). Using this strategy, it is possible to make only one error per session. Instead, after considerable training, the pigeons averaged about 7.5 errors in an 80-trial session in the Rayburn-Reeves et al. (2011) study. Despite a more efficient strategy being available to pigeons, several procedures used to try to discourage the pigeons' use of timing have not resulted in an increase in their accuracy to approximate a win-stay/lose-shift rule (Laude, Stagner, Rayburn-Reeves, \& Zentall, 2014; Rayburn-Reeves, Laude, \& Zentall, 2013; Rayburn-Reeves et al., 2011; Rayburn-Reeves \& Zentall, 2013; Stagner et al., 2013).

Contrary to the results with pigeons, rats tested on a spatial midsession reversal task using levers approximated optimal win-stay/lose-shift behavior, showing almost no anticipatory errors and very few perseverative errors (Rayburn-Reeves, Stagner, et al., 2013). Additionally, the rats continued to show a high degree of accuracy when the point of reversal in the session was pseudorandomly varied between sessions to one of five different locations, and when two or three reversals occurred during each session.

Although it is possible that the difference between rats' and pigeons' performance on this task reflects a species difference in sensitivity to the feedback from the outcome of the previous trial(s), it is also possible that the rats used the position of their leverpressing paw as a cue during the intertrial interval (Laude et al., 2014; McMillan, Kirk, \& Roberts, 2014; McMillan \& Roberts, 2015). Thus, the rats' superior performance may have stemmed from being able to retain the response from the previous trial over the course of the intertrial interval (ITI). Indeed, such mediating or precurrent behaviors during delays have been reported in delayed matching-to-sample tasks with rats using levers (see Herremans \& Hijzen, 1997, for a review). As such, being able to orient to a lever with a paw during the ITI could increase the rats' ability to use a winstay/lose-shift response rule.

Further evidence that the nature of the response contributes to the difference in accuracy between species has come from the finding that when rats were trained in a T-maze version of the midsession reversal task, they showed heightened anticipatory and perseverative errors (McMillan et al., 2014). This led the authors to argue that the rats' excellent performance with the two-lever task may have been due to their ability to use their body position as a cue during the ITI (see also Laude et al., 2014; McMillan \& Roberts, 2015). However, the possibility of using body position as a cue is not likely to be the only determinant of win-stay/lose-shift reversal accuracy, because when pigeons were given the same opportunity to use body position as a cue, by having them make a treadle-press rather than a key-peck response, they showed no improvement in their reversal accuracy (Stagner et al., 2013).

Previous research has also indicated that pigeons and rats may exhibit differences under explicit conditions attempting to train a win-stay, win-shift, lose-stay, or lose-shift contingency. For example, Randall and Zentall (1997) found that pigeons readily learned a win-stay response but struggled with a win-shift response. Furthermore, when rats were trained in a T-maze to win-stay (i.e., to return to the same arm) versus win-shift, they initially showed a bias to shift, visiting the previously unvisited arm early in training and showing reduced accuracy (Haig, Rawlins, Olton, Mead, \& Taylor, 1983); this pattern of results has been found in adult rats in a T-maze (Haig et al., 1983), as well as in young rats in a Y-maze (Gittis et al., 1988). Additionally, an experiment by Evenden and Robbins (1984), training rats in either an operant box with levers or a Y-maze, indicated that the rats in the operant chamber initially tended to stay, whereas the rats in a Y-maze showed a high level of shift behavior that changed to stay behavior with training. These results suggest that rats' response biases are flexible with training but show different initial levels of stay and shift behavior, depending on the apparatus. This result may further suggest that the comparison of rats in a T-maze to pigeons in an operant box may have more to do with the apparatus than with differences in response typographies (McMillan et al., 2014).

Although previous research aimed at improving pigeons' reversal performance with the midsession reversal task, by using spatial discriminations (Laude et al., 2014; RayburnReeves, Laude, \& Zentall, 2013) and a treadle-step response (Stagner et al., 2013) to better approximate the procedures used with rats, was not successful (but see Rayburn-Reeves, Stagner, et al., 2013), an alternative approach would be to ask whether the rats' excellent performance on the midsession reversal task depended on their ability to maintain proximity to the lever just pressed. The difference between rats' accuracy on the midsession reversal task when performed in the operant 
chamber and the T-maze suggests that the nature of the response may be an important factor in rats' performance with this task. Thus, the goal of the present research was to assess rats' performance on a midsession reversal task using two different response typographies: levers, which allow the rat to maintain proximity to the manipulandum with the paw or body during the ITI, and nose pokes, a response operant that has shown operant properties similar to levers (Schindler, Thorndike, \& Goldberg, 1993) but that, as with pigeons using key pecks, makes it difficult to maintain proximity to the manipulandum during the ITI.

\section{Method}

\section{Subjects}

Thirteen female Sprague-Dawley albino rats (Rattus norvegicus) about 12 months old originally obtained from Harlan Laboratories (Indianapolis, IN) were used as subjects. All subjects had previous experience in the lab with a probability-matching task. The rats were maintained at $85 \%$ of their target weight based on age throughout the experiment. They were singly housed in polystyrene cages with free access to water in a colony room that was maintained on a $12-\mathrm{h} / 12-\mathrm{h}$ light/dark cycle. The rats were maintained in accordance with a protocol approved by the Institutional Animal Care and Use Committee at the University of Kentucky.

\section{Apparatus}

The experiment was conducted in a standard rodent operant chamber (Med Associates, St. Albans, VT) measuring $25.7 \mathrm{~cm}$ across the response panel, $33 \mathrm{~cm}$ from ceiling to floor, and $31 \mathrm{~cm}$ from response panel to the back wall. The chamber had a house light on the front wall located $1.3 \mathrm{~cm}$ from the ceiling. A pellet dispenser delivered pellets to a food well that was centered on the response panel, $5.6 \mathrm{~cm}$ from the floor. Two retractable response levers, $3.6 \mathrm{~cm}$ wide, were located on either side of the food magazine, each located $2.3 \mathrm{~cm}$ from the wall and $5.6 \mathrm{~cm}$ from the floor. A standard nose-poke response well with a 2.54$\mathrm{cm}$-diameter entrance hole was located $3 \mathrm{~cm}$ above each lever. Reinforcement consisted of one pellet (MLab Rodent Tablet $45 \mathrm{mg}$ 1811156, TestDiet, Richmond, IN) for each correct response. The experimental chamber was located in a small isolated room to reduce extraneous visual and auditory stimulation. Med-PC IV controlled the experiment from a desktop computer located in an adjacent room (Tatham \& Zurn, 1989).

\section{Procedure}

Subjects were randomly assigned to the lever group $(n=7)$ or the nose-poke group $(n=6)$. Only the assigned response manipulandum (lever or nose-poke) was active for each rat. At the start of each trial the house light was illuminated, indicating that the two manipulanda were operable. For subjects in the nose-poke group, each trial began with the illumination of both nose-poke wells. After a response to either manipulandum, the house light extinguished and, in the nose-poke group, the well lights extinguished. If the subject responded to $\mathrm{S} 1$ (the first correct stimulus, counterbalanced as either the left or the right manipulandum), a single pellet was delivered to the food magazine at the start of the 5-s ITI. If the rat responded to $\mathrm{S} 2$ (the correct stimulus after the reversal), the house light extinguished for a 5-s ITI but no food was delivered. For subjects in the lever group, each trial began similarly to those for the nose-poke group, with the illumination of the house light. Once a response was made, the house light extinguished but the levers remained extended into the chamber. Half of the subjects in each group were assigned the right manipulandum as $\mathrm{S} 1$, and the other subjects were assigned it as $\mathrm{S} 2$. Sessions lasted 8-12 min.

The experiment consisted of three phases: a 40-trial midsession reversal, an 80-trial midsession reversal, and an 80 -trial midsession reversal with varied reversal points. For the first 20 trials of each 40-trial session, subjects were trained with $\mathrm{S} 1+/ \mathrm{S} 2-$. For the remaining trials of the session, the contingencies were reversed (S2+/S1-). Subjects were trained for 50 sessions with 40 -trial sessions. For the following 50 sessions (Sessions 51-100), the session length was extended to 80 trials per session (with the reversal occurring at Trial 41). For the final 50 sessions (Sessions 101-150), the reversal point varied between sessions, occurring in one of five locations in the session (after Trial 10, 25, 40, 55, or 70). The reversal point for each session was randomized over sessions, with each rat completing ten sessions at each reversal point.

\section{Data analysis}

The data were analyzed in blocks of five training sessions, because it was anticipated that the rats in the two groups might acquire the midsession reversal task at different rates. In addition, because different kinds of errors are likely to have different implications, we separately analyzed the different error types. First, to get an overall estimate of the rats' accuracy, we analyzed total errors. Then, because differences in the asymptotic levels of accuracy represented a measure of the rats' ability to repeat a previous correct response to either S1 or S2, we analyzed separately the errors that were made in the first 16 trials (or 36 trials, in Phase 2) of the session (global anticipatory errors) and errors that were made in the last 16 trials (or 36 trials, in Phase 2) of the session (global perseverative errors). Additionally, local measures closer to the reversal were also analyzed. We assessed the rats' use of time into the session as a cue to reverse through measuring errors made during the last five trials prior to the reversal (S2 responses on Trials 17-21 or 
Trials 37-41; local anticipatory errors), with Trial 21 reversecoded because feedback indicating that the reversal had occurred followed choice on Trial 21 (or Trial 41, in Phase 2), and measuring the rats' sensitivity after the reversal (S1 responses on Trials 22-26 or Trials 42-46; local perseverative errors). Finally, to get a more precise measure of the rats' sensitivity to the reversal on Trial 21 (or Trial 41, in Phase 2) we analyzed the drop in the rats' choices of S1 from Trials 17-21 to Trial 22 (or from Trials 37-41 to 42, in Phase 2).

For acquisition, all measures were averaged over blocks of five sessions, resulting in ten blocks of acquisition data for the 40- and 80-trial phases. The tests were analyzed using a 10 (session block) $\times 2$ (group) repeated measures analysis of variance (ANOVA). The data from the variable-reversalpoint sessions were analyzed with a 5 (reversal point) $\times 2$ (group) repeated measures ANOVA for each of the three local variables. Analyses of global anticipatory and perseverative errors were omitted for the final phase due to the differences in numbers of trials for each point of reversal. Finally, the performance of the two groups was compared on the last block of training of each phase with a 3 (phase) $\times 2$ (group) repeated measures ANOVA for each of the localized measures of performance. For the variable-point-of-reversal phase, only data from the 40-trial reversal point were used for this analysis.

All statistics were calculated using the SPSS software. Where appropriate, Greenhouse-Geisser and Levine's tests for unequal variances were used in the reported statistics. For the sake of brevity, expected main effects of block (normal acquisition functions) and nonsignificant effects are not reported. All post-hoc pairwise comparisons were analyzed with Hochberg adjustments (Hochberg, 1988).

\section{Results}

\section{0-trial phase}

Both groups showed reasonably accurate performance by the last block of ten sessions (see Fig. 1). The only apparent group difference was that the lever group appeared to show somewhat greater S1 choices following the reversal. However, the two groups showed different patterns of acquisition.

Global measures Global measures of accuracy are presented in blocks of five sessions on the left side of Fig. 2. We found a significant effect of session block for each of the three kinds of global errors: total errors (top left), anticipatory errors (middle left), and perseverative errors (bottom left). Additionally, the lever group showed significantly fewer total errors $(M=11.41, S E M=1.27,95 \%$ CI $[9.10,13.72])$ than the nose-poke group $(M=16.3, S E M=1.77,95 \%$ CI [14.16, $18.44]), F(1,11)=11.68, p=.006, \eta_{\mathrm{p}}{ }^{2}=.52,95 \%$ CI [1.74, $8.04]$, significantly fewer anticipatory errors (Lever: $M=$



Fig. 1 Mean trial-by-trial percentage choices of S1 over the last block of ten sessions in the 40-trial phase

10.42, $S E M=2.02,95 \%$ CI [7.94, 12.90]; Nose-poke: $M=$ $14.04, S E M=2.16,95 \%$ CI $[11.74,16.33]), F(1,11)=5.56, p$ $=.038, \eta_{\mathrm{p}}{ }^{2}=.336,95 \%$ CI $[0.24,7.00]$, and significantly fewer perseverative errors (Lever: $M=3.83, S E M=1.34$, $95 \%$ CI [0.04, 7.63]; Nose-poke: $M=12.94, S E=3.07$, $95 \%$ CI $[9.42,16.46]), F(1,11)=14.99 p=.003, \eta_{\mathrm{p}}{ }^{2}=.58$, $95 \%$ CI $[3.93,14.28]$, indicating better global performance for the lever group across acquisition.

Local measures For the localized measures of performance (Fig. 2 right), only local perseverative errors (bottom right) and the drop in $\mathrm{S} 1$ responding (top right) showed significant effects of session block. Also, a significant Block $\times$ Group interaction was apparent in the drop in $\mathrm{S} 1$ responding between Trials 21 and 22, $F(9,99)=2.59, p=.01, \eta_{\mathrm{p}}{ }^{2}=.19$, As can be seen in Fig. 2 (top right) the interaction can be attributed largely to differences between the groups in the last two 5session blocks of training. Local anticipatory errors yielded no effect of block or group (Fig. 2, middle right), indicating that these error types did not improve across acquisition. There were no significant group differences.

Last block of training Steady-state group differences (data pooled over the last ten sessions of training) were assessed for each of the six variables. The results showed that the nose-poke group showed significantly greater perseverative errors $(M=$ $4.9, S E M=0.74,95 \%$ CI $[2.50,7.29]), t(11)=2.75, p=$ $.019,95 \%$ CI $[0.69,6.25]$, and a greater drop in $\mathrm{S} 1$ responding $(M=45.14, S E M=4.3,95 \%$ CI $[34.63,55.65])$ than did the lever group $(M=1.43, S E M=0.98,95 \% \mathrm{CI}[-0.47,3.32]$, and $M=21, S E M=3.64,95 \%$ CI [11.64, 30.36], respectively), $t(11)$ $=4.2, p=.001,95 \%$ CI [11.50, 36.79]. These results indicate that the nose-poke group showed greater sensitivity to switching after the reversal, but that the lever group ultimately maintained higher accuracy levels in the second half of the session.

\section{0-trial phase}

For the 80-trial phase, the only apparent group difference was that the lever group appeared to show fewer anticipatory errors 

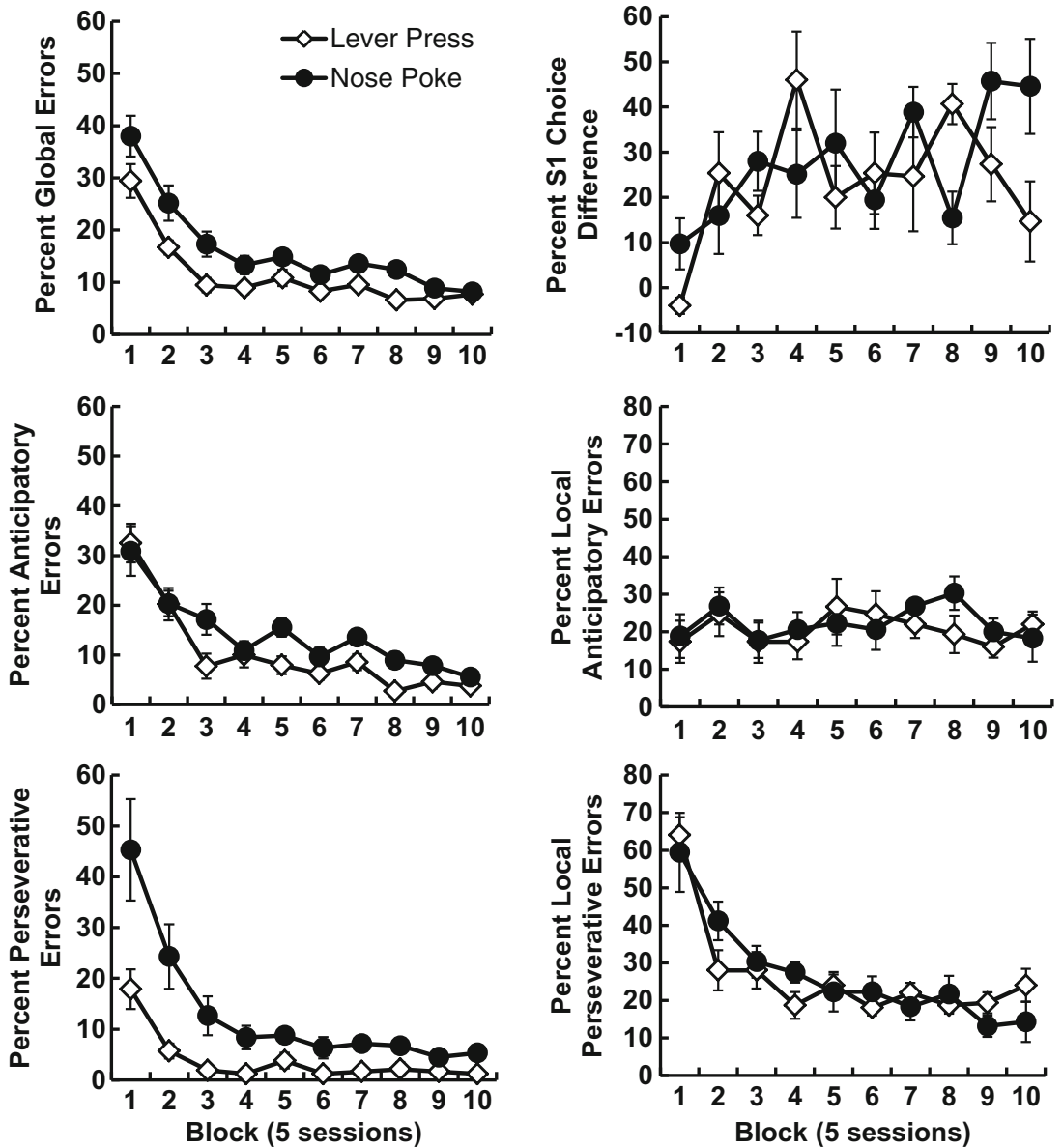

Fig. 2 40-trial phase: Mean ( $\pm S E M)$ percentage errors made over blocks of training for global errors (top left), anticipatory errors (middle left), perseverative errors (bottom left), the S1 drop in errors after the first

and a smaller drop in S1 responding following the first reversal trial (see Fig. 3).

Global measures The six variables were again computed for this phase, and the results are presented in Fig. 4. We observed significant effects of session block for global errors (top left), anticipatory errors (middle left), and perseverative errors (bottom left). Finally, there were significantly more global errors, $F(1,11)=6.91, p=.023, \eta_{\mathrm{p}}{ }^{2}=.39,95 \% \mathrm{CI}[0.29,3.23]$, and

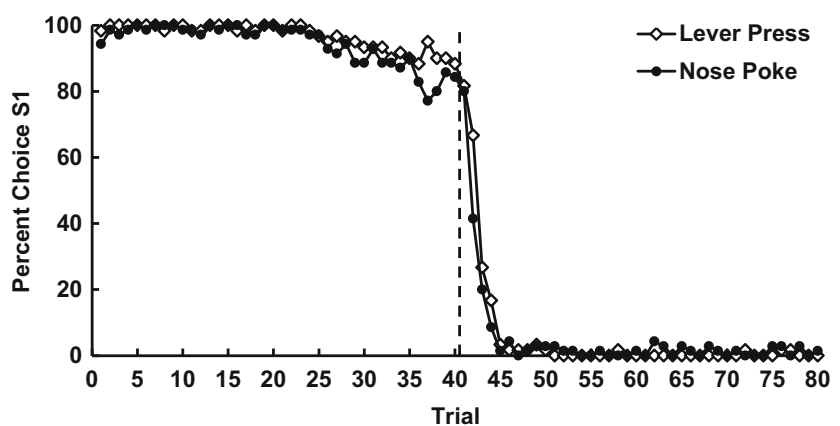

Fig. 3 Mean trial-by-trial percentage choices of S1 over the last block of ten sessions in the 80 -trial phase

reversal trial (top right), local anticipatory errors (middle right), and local perseverative errors (bottom right) over training

perseverative errors, $F(1,11)=5.84, p=.034, \eta_{\mathrm{p}}{ }^{2}=.35,95 \%$ CI $[0.13,2.81]$, for the nose-poke group $(M=6.17$, SEM $=$ $0.84,95 \%$ CI $[5.17,7.17] ; M=2.46, S E M=0.78,95 \%$ CI $[1.55,3.37])$ than for the lever group $(M=4.41, S E M=0.8$, $95 \%$ CI $[3.33,5.49] ; M=0.99, S E M=0.5,95 \%$ CI [0.01, 1.97]), indicating that the lever group acquired the task faster than the nose-poke group.

Local measures For the local measures of performance (Fig. 4, right), we found no significant effects of block, indicating that performance around the reversal point did not change significantly across blocks of training. The lever group did, however, make significantly fewer local anticipatory errors $(M=10.8, S E M=$ $4.54,95 \%$ CI $[6.73,14.87]), F(1,11)=8.04, p=.016, \eta_{\mathrm{p}}{ }^{2}=$ $.422,95 \%$ CI [1.60, 12.69], but also more local perseverative errors $(M=31.44, S E M=5.23,95 \%$ CI $[24.29,34.65]), F(1,11)$ $=10.25, p=.008, \eta_{\mathrm{p}}{ }^{2}=.48,95 \% \mathrm{CI}[3.21,17.32]$, than the nosepoke group $(M=17.94, S E M=4.55,95 \% \mathrm{CI}[14.18,21.71] ; M$ $=19.2, S E M=3.9,95 \% \mathrm{CI}[14.41,24.0])$. This pattern of results suggests that the lever group was able to maintain S1 responding prior to the reversal, but that the nose-poke group had a tendency to switch to S2 responding more quickly following the reversal. 

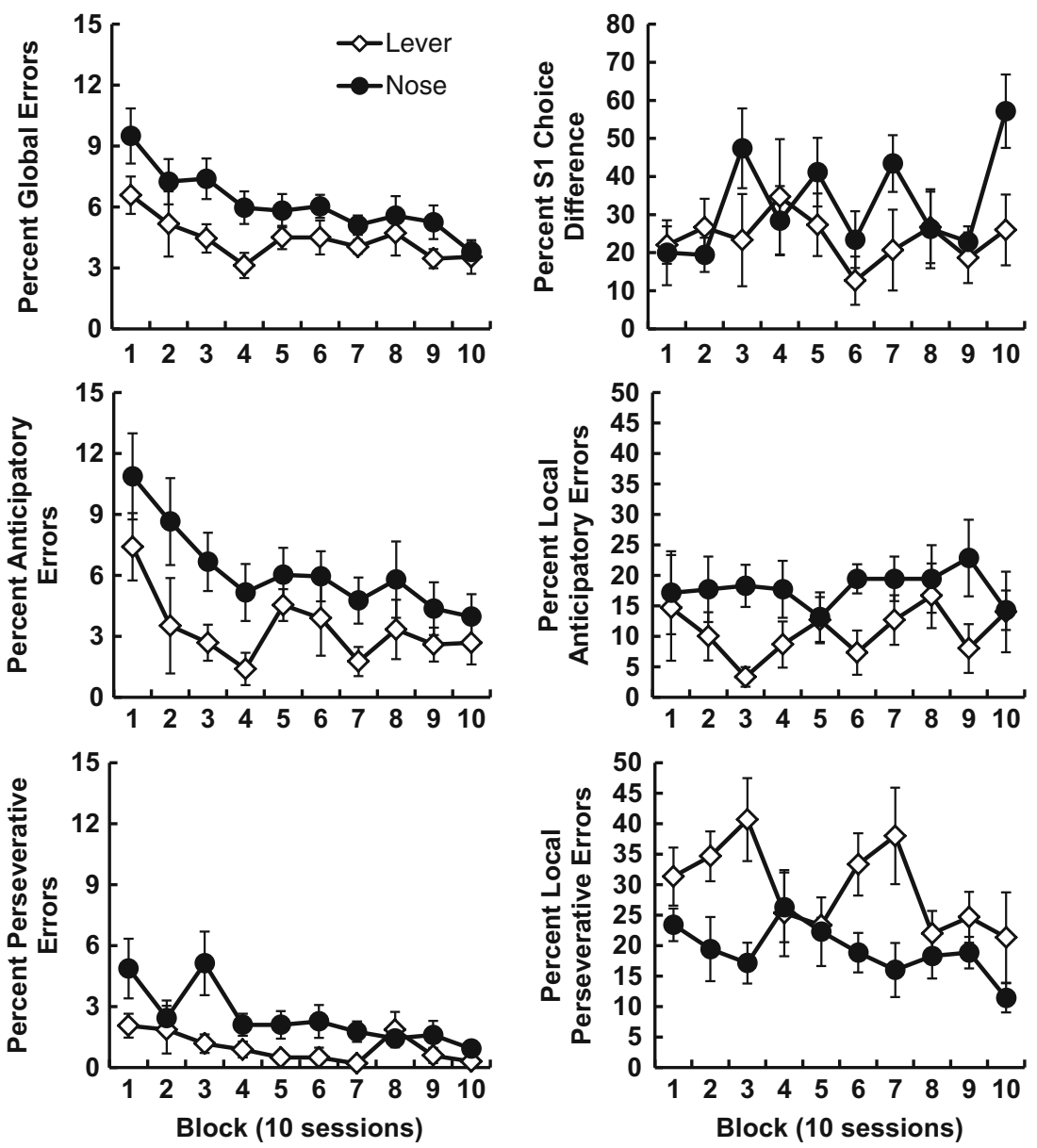

Fig. 4 80-trial phase: Mean $( \pm S E M)$ percentage errors made over blocks of training for global errors (top left), anticipatory errors (middle left), perseverative errors (bottom left), the S1 drop in errors after the first

reversal trial (top right), local anticipatory errors (middle right), and local perseverative errors (bottom right) over training

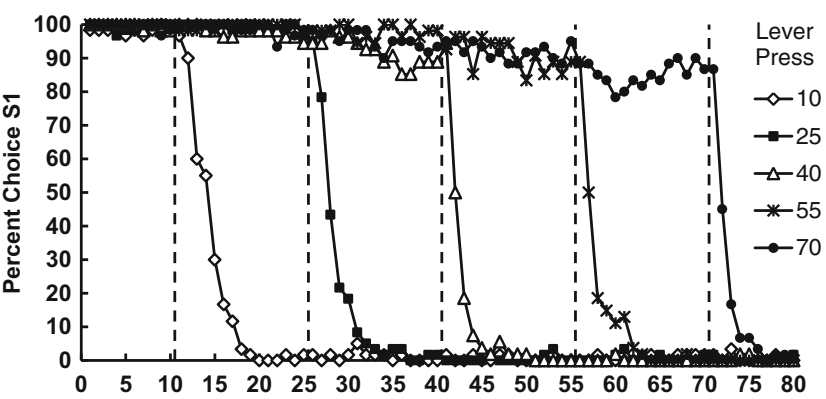

\section{Varied point of reversal}

Because only ten sessions were conducted for each of the points of reversal, the data were pooled for purposes of this analysis. Overall, the rats tended to show greater anticipatory errors when the reversal was late, and greater perseverative errors when it was early. The average performance of each group at each reversal point pooled over blocks is presented in Fig. 5. Because the group differences were minimal (see Fig. 6 top), choices of S1 for both groups combined, on the five trials before the reversal and the five trials after the reversal, are presented in Fig. 6 (bottom).

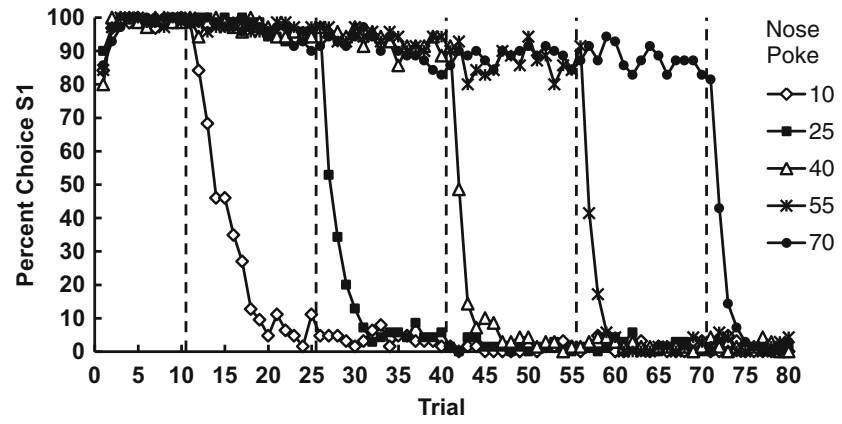

Fig. 5 Mean percentage trial-by-trial choices of S1 at each reversal point over ten sessions for the lever group (top) and the nose-poke group (bottom) in the varied-reversal phase 

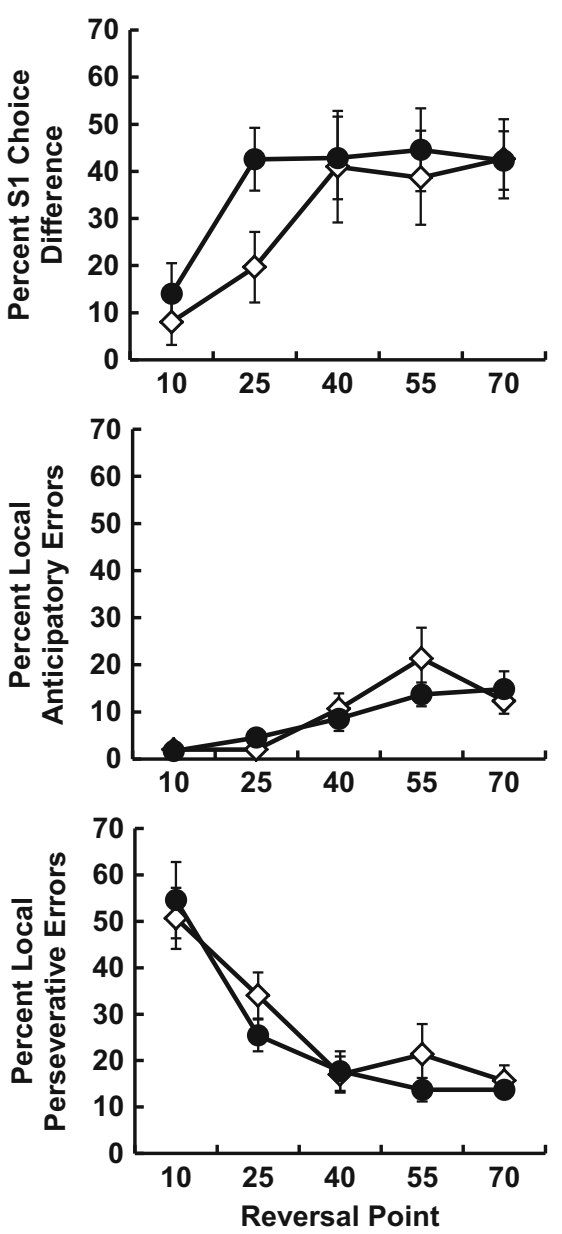

Fig. 6 Mean percentage choices of S1, relative to the reversal, of the lever and nose-poke groups combined over reversal points (top), and mean percentage choices of S1 for each reversal point pooled over groups (bottom) in the varied-reversal phase

Local measures For the local measures of performance (Fig. 7), significant differences in performance between the reversal points were found for local anticipatory errors (middle), $F(4,44)=10.44, p<.001, \eta_{\mathrm{p}}{ }^{2}=.49$; local perseverative errors (bottom), $F(4,44)=34.52, p<.001, \eta_{\mathrm{p}}{ }^{2}=.76$; and the drop in $\mathrm{S} 1$ responding on Trial 42, $F(4,44)=9.07, p<.001$, $\eta_{\mathrm{p}}{ }^{2}=.45$ (top). Generally, anticipatory errors and sensitivity to the reversal (the difference between choices of S1 on Trials 41 and 42) increased as the point of reversal occurred later in the session, whereas perseverative errors decreased, but no significant group differences were apparent.

\section{Between-phase comparisons}

Between-phase comparisons for the local measures of behavior can be seen in Fig. 8, which shows that the groups differed in the numbers of errors made in Phases 1 and 2, but not in Phase 3. For local anticipatory errors (top), there was a significant effect of phase, $F(2,22)=5.56, p=.011, \eta_{\mathrm{p}}{ }^{2}=.34$, as
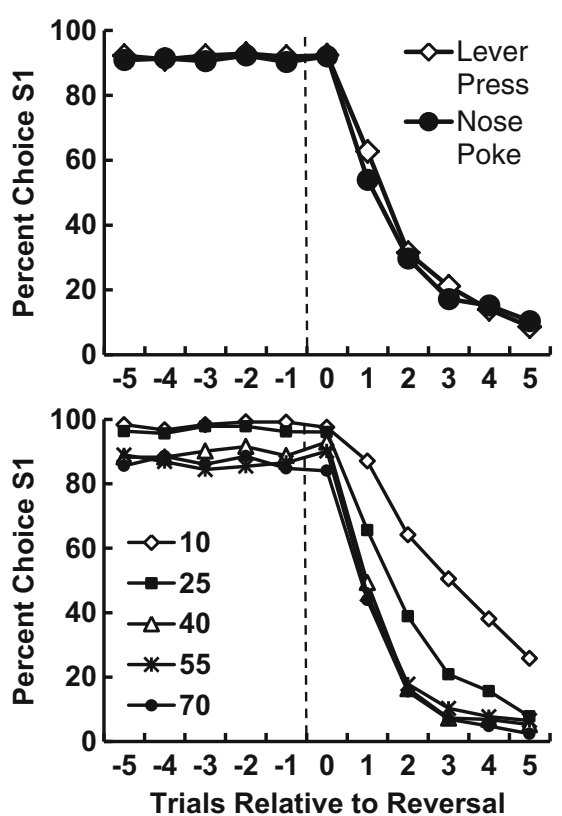

Fig. 7 Mean $( \pm S E M)$ percentage errors made at each reversal point for the drop in S1 choices from Trial 41 to 42 (top), local anticipatory errors (middle), and local perseverative errors (bottom) training in the variedreversal phase

well as a significant decreasing linear trend, $F(1,11)=17.23$, $p=.002, \eta_{\mathrm{p}}{ }^{2}=.61$, with post-hoc analyses showing that the varied phase had significantly fewer local anticipatory errors than the 40 -trial phase, $t(11)=4.15, p=.005,95 \% \mathrm{CI}[2.28$, 18.77], indicating a reduction in errors after extended training. Local perseverative errors (middle) showed no significant effects of phase or group. For the drop in S1 responding on the trial following the reversal (bottom), analyses showed no effect of phase, but did show a significant group effect, $F(1,11)$ $=6.69, p=.025, \eta_{\mathrm{p}}{ }^{2}=.38,95 \%$ CI $[2.54,39.39]$, indicating that the nose-poke group showed greater sensitivity to the reversal than did the lever group, consistent with the previous measures.

\section{Discussion}

In the present experiment, we sought to assess the effects of the nature of rats' responses on performance of the midsession reversal task. Specifically, it was hypothesized that the rats' superior performance on this task (Rayburn-Reeves, Stagner, et al., 2013) may have been due to the fact that the rats pressed the lever with their paw rather than made the response with their nose, potentially allowing the lever rats to better bridge the 5-s ITI (see, e.g., Laude et al., 2014; McMillan et al., 2014). To test this hypothesis, we included a group for which the response consisted of a nose-poke, which more closely resembled the pigeons' key peck. This response required the rats to use the same body part to make their response as they 



Fig. 8 Mean $( \pm S E M)$ percentage errors for the last block of training in the 40 -trial phase, the 80 -trial phase, and at the 40 -trial reversal point of the varied-reversal phase for local anticipatory errors (top), local perseverative errors (middle), and the drop in S1 choices on the trial following the first reversal trial (bottom)

used for eating, reducing the ability to use body orienting as a cue to which response to make on the next trial.

The results indicated that, overall, the nose-poke group tended to make more errors than the lever group during acquisition. In particular, the nose-poke group made significantly more global errors of all types in the 40-trial phase, as well as more global, perseverative, and local anticipatory errors in the 80-trial phase. Together, these results support the notion that the advantage of body-orienting cues in the lever group may produce faster learning in this task. However, by the last block of training both groups showed high degrees of accuracy. As such, although the lever group made fewer local anticipatory errors in the 80-trial phase, the nose-poke group made fewer local perseverative errors during acquisition in the 40-trial phase, and showed a greater drop in $\mathrm{S} 1$ responding on the trial following the reversal in both the 40- and 80-trial phases. Thus, the results suggest that the lever group's faster acquisition did not result in higher levels of asymptotic performance and that, although they showed better performance both globally and locally prior to the reversal, the nose-poke group showed better performance locally following the reversal.
The better performance by the nose-poke group locally after the reversal was surprising, given the lever group's superior performance on the other measures. However, assuming that the lever rats were able to mediate the ITI by being better able to separate the lever response from the consummatory response, the present results suggest that the response separation (paw vs. head) can help reduce overall errors but may also result in more errors immediately following the reversal. That is, if the rat responds with its paw to the $\mathrm{S} 1$ lever immediately after eating from the feeder, a repetitive behavior pattern may develop that leads to better overall accuracy but that also may be more difficult to adjust immediately following the reversal. It is not obvious, however, why a similar behavior pattern would facilitate the reversal for pigeons (Rayburn-Reeves, Stagner, et al., 2013) but not for the rats in the present experiment. The difference is possibly due to differences in the saliences of reinforcement between a raised feeder for the pigeons and a dispensed pellet for the rats, or to the fact that the rats can leave their paw on or near the lever. In contrast, the pigeons had to leave the previously chosen stimulus to eat and then return to it, more closely resembling the nose-poke group, which also showed relatively greater sensitivity to the reversal. Thus, although use of the lever response may facilitate overall accuracy during acquisition, it does not suggest greater flexibility immediately following the reversal.

Examination of the data from the variable-point-of-reversal sessions also suggests that, as with pigeons (Rayburn-Reeves et al., 2011), later points of reversal tended to result in more anticipatory errors, whereas earlier points of reversal tended to result in greater perseverative errors. Additionally, in the variable-point-of-reversal sessions, both groups showed similar levels of performance, a result further supporting the conclusion that sufficient training reduces the performance differences seen early between the lever and nose-poke groups.

The pattern of results found in the present experiment also suggests that anticipatory and perseverative errors may be attributable to distinctive mechanisms. Although global and local perseverative errors declined with training, local anticipatory errors did not. This result may have occurred for several reasons, one of which may arise from two competing tendencies. The first is that learning that $\mathrm{S} 1$ responses will be reinforced during the first half of each session generally improves with training, leading to better overall accuracy. Conversely, the rats were also learning to time the interval to the reversal, potentially increasing the tendency to respond to S2 prior to the reversal, due to inaccuracies in timing. These two competing tendencies may have then resulted in smaller improvements in local anticipatory errors that were only apparent after extensive experience in Phase 3. More importantly, a potentially large difference exists between anticipatory and perseverative errors: For anticipatory errors, there is an ambiguity in the feedback that one can obtain from that error type. Although it is an error, the same response could be 
correct on the next trial, a feature that is not present after the reversal with perseverative errors. That is, a perseverative error provides information about what response will be correct on the next trial.

McMillan and Roberts (2015) also recently suggested that, because temporal cues are intrinsic and always available to the animal, they may be relied upon in the absence of other sources of information, such as when memory for a preceding response and its outcome fails. It is also possible that temporal cues change in their salience over the course of the session, becoming more ambiguous as the current trial comes closer to the reversal. Additionally, as anticipatory errors increase, memory for the preceding response and its outcome may also increase ambiguity in this source of information, increasing the animals' reliance on temporal cues when approaching the time of the reversal (even though the time to the reversal may also be ambiguous). However, for perseverative errors, the ambiguity of the outcome of the previous trial as a cue is reduced as the trial progresses further from the reversal. This account, then, ostensibly pits two competing sources of information against each other: external feedback from the previous trial, and intrinsic temporal cues. Indeed, some evidence that memory for the preceding response may be involved is suggested by the fact that pigeons' performance increases when both spatial and visual information are relevant (McMillan et al., 2014; McMillan \& Roberts, 2012). If this is the case, then the results from the present experiment showing differences between pigeons and rats may be due to differences in memory for preceding events and/or differences in the salience of temporal cues.

Additionally, although the lever group in the present experiment performed the midsession reversal significantly better in acquisition, and the nose-poke rats showed better performance immediately after the reversal late in training, their reversal accuracies on this task did not approach the near win-stay/ lose-shift performance of rats trained with a similar lever discrimination reversal in the Rayburn-Reeves, Stagner, et al. (2013) study. The only notable difference between our levergroup rats and their rats was the sex of the rats: We used female Sprague-Dawley rats, and the Rayburn-Reeves, Stagner, et al. used male Sprague-Dawley rats. Although little published evidence from reversal studies has indicated that female rats may perform worse than male rats, a recent meta-analysis reported significantly better performance by male rats relative to females in spatial cognition tasks such as the Morris water maze and the radial maze (Jonasson, 2005). Female rats have also shown greater locomotor activity in a variety of novel environments at a young age, possibly attending less to the task (Hyde \& Jerussi, 1983; Lynn \& Brown, 2009). Consistent with this hypothesis, RayburnReeves, Stagner, et al. found poorer performance by pigeons that moved away from the response keys during the ITI, suggesting that increased locomotor activity in the apparatus may account for the difference in findings between the RayburnReeves, Stagner, et al. study and the results of the lever group from the present experiment. Alternatively, this increased activity or a decreased memory for preceding events could also produce a greater reliance on temporal information by female than by male rats; however, future research will be needed to test these possibilities.

This possible sex difference also may warrant a qualitative comparison of the rats performing a spatial discrimination reversal with the nose-poke response and a 5-s ITI with pigeons performing a similar spatial discrimination using a keypeck response (Laude et al., 2014; Rayburn-Reeves, Laude, \& Zentall, 2013). With an 80-trial session in the present experiment, the global accuracies on the last training block were $97 \%$ and $95 \%$ for the lever and nose-poke rats, respectively, whereas global accuracy for the pigeons with a 5-s ITI spatial discrimination and a comparable amount of training was $89 \%$ (Laude et al., 2014; Rayburn-Reeves, Laude, \& Zentall, 2013). However, both groups of female rats in the present study appeared to be qualitatively similar to the pigeons from the McMillan et al. (2014) study in which spatial cues were relevant and color cues were redundant (e.g., green was always on the left) on a varied point of reversal with a 4-s ITI. In the present experiment, the global accuracies were $95 \%$, $96 \%, 96 \%, 95 \%$, and $92 \%$ with the reversal points after Trials $10,25,40,55$, and 70 for the lever group, and $93 \%$, $95 \%, 95 \%, 93 \%$, and $91 \%$ for the nose-poke group (with a 5-s ITI), respectively. For the pigeons from McMillan et al.'s study, the global accuracies were $96 \%, 97 \%, 96 \%, 94 \%$, and $89 \%$ with twice as much training. Thus, pigeons performing a redundant spatial/visual reversal with varied points of reversal may be similar to female Sprague-Dawley rats, possibly suggesting quantitative differences in the capacities to use different sources of information.

The accuracy of rats in the present experiment was also superior to that of rats in McMillan et al. (2014) that performed a spatial discrimination in a T-maze. It is difficult to compare the accuracies of rats in a T-maze with those in an operant box, because the rats are unable to use orienting cues to facilitate bridging the ITI in a T-maze. Because this task requires memory for the last response and its consequence, the T-maze procedure may be disruptive, because it involves moving the animal from the goal box to the start box between trials and involves a longer delay between the choice response and reinforcement (see Evenden \& Robbins, 1984; Gittis et al., 1988; Haig et al., 1983). Thus, the present comparison of rats responding with a nose-poke in an operant chamber offers the most similar comparison to pigeons pecking.

The present study contributes several novel findings. First, it shows that the lever group's ability to bridge the ITI to enhance memory for the response that was previously rewarded appears to contribute to rats' accuracy in acquisition. However, this advantage may not have been facilitative 
locally around the reversal, because the nose-poke rats were able to achieve a similar level of reversal accuracy after sufficient training. Furthermore, the nose-poke rats showed a more rapid response to the feedback from the reversal. In the future, observation of rats would be useful to assess these potential response-produced cues. Additionally, in the present study we found a potential sex difference in Sprague-Dawley rats, although the mechanism responsible for this difference is not yet clear, since we did not have a male group for comparison (see Rayburn-Reeves, Stagner, et al., 2013). Most importantly, in the present experiment we found asymmetries in the acquisition of different components of the reversal task, indicating the need for future studies to more thoroughly quantify and separate global and local performance around the reversal point. Finally, the ability to orient to the lever does appear to facilitate acquisition of the midsession reversal in rats, but it does not account for the difference in performance between rats and pigeons with this task.

\section{References}

Bitterman, M. E. (1965). Phyletic differences in learning. American Psychologist, 20, 396-410. doi:10.1037/h0022328

Braggio, S. M., Braggio, J. T., Cochran, T. C., \& Ellen, P. (1983). Discrimination-reversal learning of normal and septal rats. Psychological Reports, 53, 647-654.

Evenden, J. L., \& Robbins, T. W. (1984). Win-stay behaviour in the rat. Quarterly Journal of Experimental Psychology, 36B, 1-26. doi:10. 1080/14640748408402190

Gittis, A., Stark, H., Arnold, C., Geter, B., Frazier, D., \& Olton, D. (1988). Emergence of choice strategies in the rat: Shift-stay differentiation precedes win-lose differentiation. Animal Learning \& Behavior, 16, 15-18. doi:10.3758/BF03209038

Haig, K. A., Rawlins, J. N. P., Olton, D. S., Mead, A., \& Taylor, B. (1983). Food searching strategies of rats: Variables affecting the relative strength of stay and shift strategies. Journal of Experimental Psychology: Animal Behavior Processes, 9, 337-348.

Harlow, H. F. (1950). Performance of catarrhine monkeys on a series of discrimination reversal problems. Journal of Comparative and Physiological Psychology, 43, 231-239.

Herremans, A. H. J., \& Hijzen, T. H. (1997). The delayed-conditionaldiscrimination task improves measurement of working memory in rats. Neuroscience \& Biobehavioral Reviews, 21, 371-379.

Hochberg, Y. (1988). A sharper Bonferroni procedure for multiple tests of significance. Biometrika, 75, 800-802.

Hyde, J. F., \& Jerussi, T. P. (1983). Sexual dimorphism in rats with respect to locomotor activity and circling behavior. Pharmacology Biochemistry and Behavior, 18, 725-729. doi:10.1016/00913057(83)90014-X

Izquierdo, A., \& Jentsch, J. D. (2012). Reversal learning as a measure of impulsive and compulsive behavior in addictions. Psychopharmacology, 219, 607-620. doi:10.1007/s00213-011-2579-7

Jonasson, Z. (2005). Meta-analysis of sex differences in rodent models of learning and memory: A review of behavioral and biological data.
Neuroscience \& Biobehavioral Reviews, 28, 811-825. doi:10.1016/ j.neubiorev.2004.10.006

Laude, J. R., Stagner, J. P., Rayburn-Reeves, R., \& Zentall, T. R. (2014). Midsession reversals with pigeons: Visual versus spatial discriminations and the intertrial interval. Learning \& Behavior, 42, 40-46. doi:10.3758/s13420-013-0122-x

Lynn, D. A., \& Brown, G. R. (2009). The ontogeny of exploratory behavior in male and female adolescent rats (Rattus norvegicus). Developmental Psychobiology, 51, 513-520. doi:10.1002/dev. 20386

McMillan, N., Kirk, C. R., \& Roberts, W. A. (2014). Pigeon (Columba livia) and rat (Rattus norvegicus) performance in the midsession reversal procedure depends upon cue dimensionality. Journal of Comparative Psychology, 128, 357-366. doi:10.1037/a0036562

McMillan, N., \& Roberts, W. A. (2012). Pigeons make errors as a result of interval timing in a visual, but not a visual-spatial, midsession reversal task. Journal of Experimental Psychology: Animal Behavior Processes, 38, 440-445. doi:10.1037/a0030192

McMillan, N., \& Roberts, W. A. (2015). A three-stimulus midsession reversal task in pigeons with visual and spatial discriminative stimuli. Animal Cognition, 18, 373-383. doi:10.1007/s10071-014-08082

Platt, J. R., \& Davis, E. R. (1983). Bisection of temporal intervals by pigeons. Journal of Experimental Psychology: Animal Behavior Processes, 9, 160-170. doi:10.1037/0097-7403.9.2.160

Rajalakshmi, R., \& Jeeves, M. A. (1965). The relative difficulty of reversal learning (reversal index) as a basis of behavioural comparisons. Animal Behaviour, 13, 203-211. doi:10.1016/0003-3472(65)90035-

Randall, C. K., \& Zentall, T. R. (1997). Win-stay/lose-shift and winshift/lose-stay learning by pigeons in the absence of overt response mediation. Behavioural Processes, 41, 227-236. doi:10.1016/ S0376-6357(97)00048-X

Rayburn-Reeves, R. M., Laude, J. R., \& Zentall, T. R. (2013a). Pigeons show near-optimal win-stay/lose-shift performance on a simultaneous-discrimination, midsession reversal task with short intertrial intervals. Behavioural Processes, 92, 65-70.

Rayburn-Reeves, R. M., Molet, M., \& Zentall, T. R. (2011). Simultaneous discrimination reversal learning in pigeons and humans: Anticipatory and perseverative errors. Learning \& Behavior, 39, 125-137. 10.3758/s13420-010-0011-5.

Rayburn-Reeves, R. M., Stagner, J. P., Kirk, C. R., \& Zentall, T. R. (2013b). Reversal learning in rats (Rattus norvegicus) and pigeons (Columba livia): Qualitative differences in behavioral flexibility. Journal of Comparative Psychology, 127, 202-211. doi:10.1037/ a0026311

Rayburn-Reeves, R. M., \& Zentall, T. R. (2013). Pigeons' use of cues in a repeated five-trial-sequence, single-reversal task. Learning \& Behavior, 41, 138-147.

Schindler, C. W., Thorndike, E. B., \& Goldberg, S. R. (1993). Acquisition of a nose-poke response in rats as an operant. Bulletin of the Psychonomic Society, 31, 291-294. doi:10.3758/BF03334932

Shettleworth, S. J. (2010). Cognition, evolution, and behavior (2nd ed.). New York, NY, US: Oxford University Press.

Stagner, J. P., Michler, D. M., Rayburn-Reeves, R. M., Laude, J. R., \& Zentall, T. R. (2013). Midsession reversal learning: Why do pigeons anticipate and perseverate? Learning \& Behavior, 41, 54-60. doi:10. 3758/s13420-012-0077-3

Tatham, T. A., \& Zurn, K. R. (1989). The MED-PC experimental apparatus programming system. Behavior Research Methods, Instruments, \& Computers, 21, 294-302. doi:10.3758/BF03205598 